\title{
A semi-empirical study of the mass distribution of horizontal branch stars in M 3 (NGC 5272)
}

\author{
A. A. R. Valcarce and M. Catelan
}

Pontificia Universidad Católica de Chile, Departamento de Astronomía y Astrofísica, Av. Vicuña Mackena 4860, $782-0436$ Macul, Santiago, Chile

e-mail: [avalcarc;mcatelan]@astro.puc.cl

Received 6 July 2007 / Accepted 19 May 2008

\begin{abstract}
Aims. Horizontal branch (HB) stars in globular clusters offer us a probe of the mass loss mechanisms taking place in red giants. For M 3 (NGC 5272), in particular, different shapes for the HB mass distribution have been suggested in the literature, including Gaussian and sharply bimodal alternatives. Here, we study the mass distribution of HB stars in M 3 by comparing evolutionary tracks for a suitable chemical composition with photometric observations.

Methods. Our approach is thus of a semi-empirical nature, describing a mass distribution favored from the standpoint of canonical stellar evolutionary predictions for the distribution of stars across the color-magnitude diagram. More specifically, we locate, for each individual HB star in M3, the evolutionary track whose distance from the star's observed color and magnitude is a minimum. We carry out tests that reveal that our method would be able to detect a bimodal mass distribution resembling that previously suggested in the literature, if present. We also study the impact of different procedures for taking into account the evolutionary speed, and conclude that they have only a small effect on the inferred mass distribution.

Results. We find that a Gaussian shape, though providing a reasonable first approximation, fails to account for the detailed shape of M3's HB mass distribution. Indeed, this mass distribution may have skewness and kurtosis that deviate slightly from a perfectly Gaussian solution. Alternatively, the excess of stars towards the wings of the distribution may also be accounted for in terms of a bimodal distribution in which both the low- and the high-mass modes are normal, the former being significantly wider than the latter. Conclusions. We also show that the inferred distribution of evolutionary times is inconsistent with theoretical expectations. This result is confirmed on the basis of three independent sets of $\mathrm{HB}$ models, suggesting that the latter underestimate the effects of evolution away from the zero-age $\mathrm{HB}$, and warning against considering our inferred mass distribution as definitive.
\end{abstract}

Key words. stars: horizontal-branch - Galaxy: globular clusters: individual: M 3 - stars: evolution

\section{Introduction}

NGC 5272 (M3) is one of the best studied globular clusters in our galaxy. Extensive photometric studies of the cluster focusing on its variable stars were published as early as the beginning of the last century (Bailey 1913), and in spite of the many searches for stellar variability in the cluster field that have been carried out since (e.g., Roberts \& Sandage 1955; Szeidl 1973; Kaluzny et al. 1998; Corwin \& Carney 2001), until very recently previously unknown variable stars in the cluster field have continued to be reported in the literature (Clementini et al. 2004).

Among M3's properties, of particular interest is its early classification, in terms of its RR Lyrae population, as an Oosterhoff $(1939,1944)$ type I (OoI) cluster. In fact, M 3 is often used to define the properties of the OoI class (e.g., Smith 1995, and references therein). In spite of its role as the prototypical OoI cluster and its extensively studied properties, theoretical interpretation of the pulsation status of M3's RR Lyrae variables remains the subject of some controversy. In particular, Rood \& Crocker (1989) and Catelan (2004) have both called attention to the fact that canonical stellar evolution/pulsation theory appears incapable of accounting for the sharply peaked distribution of (fundamentalized) periods that is found in the cluster (and possibly in other clusters as well). In particular, the Monte Carlo simulations presented by Catelan (2004), in which normal deviates were assumed (after Rood \& Crocker, who advocated a normal distribution for the masses of horizontal branch (HB) stars in M3), predicted period distributions that were most often much flatter than observed. Among the possible solutions to the problem, these authors discuss the possibility of pulsationinduced mass loss leading to "trapping" of HB stars at some point in their evolution, as well as a rather peculiar multimodal mass distribution (see Sect. 7 in Catelan 2004).

Following up on the latter scenario, Castellani et al. (2005) argued, again on the basis of Monte Carlo simulations, that a sharply bimodal mass distribution may indeed be able to account for the observed period distribution. The shapes of their two "period distribution-based" mass modes turned out to be rather peculiar. More specifically, their mode responsible for the blue HB stars is essentially flat (between about $0.61 M_{\odot}$ and $0.65 M_{\odot}$ ), while the higher-mass mode responsible for the RR Lyrae and red HB stars corresponds to a Gaussian distribution with $\langle M\rangle=0.68 M_{\odot}$ and $\sigma_{M}=0.005 M_{\odot}$. Thus their suggested low- and high-mass modes are clearly detached from one another. Note, in addition, that Castellani et al. also advocate truncating the high-mass Gaussian mode at its mean mass value (i.e., retaining only the low-mass half of the Gaussian), in order to avoid the overproduction of red HB stars.

As is well known, the evolution of an HB star, for a given chemical composition, is primarily determined by its mass (or, more precisely, by the ratio between the envelope mass and the core mass; e.g., Caloi et al. 1978). Therefore, in the case of 
relatively simple populations as monometallic globular clusters (such as M3 itself; e.g., Sneden et al. 2004), one expects, within the canonical framework, the detailed HB stellar distribution on the color-magnitude diagram (CMD) to reflect the underlying HB mass distribution. Accordingly, it should be possible, by careful examination of the available photometric data in such an extensively studied cluster as M3, to derive constraints on the shape of the underlying HB mass distribution. This is of relevance in the present context, since - as already discussed - the HB unimodality that was adopted as a working hypothesis in the HB simulations computed by Rood \& Crocker (1989) and Catelan (2004) has been identified as a possible explanation for the conflict between observed and predicted RR Lyrae period distributions in M3. In this sense, the main purpose of this paper is to check whether a multimodal mass distribution, as discussed by Catelan (2004), or a very peculiar bimodal mass distribution, as advanced by Castellani et al. (2005), is supported by the available CMD data.

Unfortunately, not many studies have attempted to derive a mass distribution for M3 on the basis of the available CMDs. While a normal distribution was favored by Rood \& Crocker (1989), and while other authors have succeeded in reproducing several different HB morphology parameters of the cluster without the need to invoke a multimodal mass distribution (e.g., Lee et al. 1990; Catelan et al. 2001b; Catelan 2004), data of much higher quality have become available in the literature since the late-80s for both variable and non-variable stars (e.g., Ferraro et al. 1997; Corwin \& Carney 2001), thus warranting a more detailed look into the photometric evidence for bimodality (or lack thereof) along the M3 HB. This is the main goal of the present paper.

In Sect. 2 we present the empirical data that were used in our study, along with the theoretical evolutionary tracks that were employed. In Sect. 3 we describe the method we used, along with tests that demonstrate that this method would be able to detect a bimodal mass distribution resembling the one suggested in the literature, if one were indeed present. In Sect. 4 we present the main results of our study. We close in Sect. 5 by presenting a summary and conclusions.

\section{Data}

\subsection{Observational data}

In order to reliably derive the mass distribution along the M $3 \mathrm{HB}$, we need a photometric database containing both variable and non-variable stars. The main problem we face is related to the fact that, in order to derive reliable mean magnitudes and colors for the RR Lyrae stars, one needs time-series photometry covering several days, and often weeks or months (particularly for variables with periods close to $0.5 \mathrm{~d}$ ), whereas non-variable red and blue HB stars can be much more straightforwardly measured. Fortunately, and as already stated (Sect. 1), the M 3 variables have been extensively studied in the literature, and reliable quantities for their "equivalent static stars" have recently been provided by Cacciari et al. (2005).

For the variable stars, we have used the Cacciari et al. (2005) database, which provides intensity-averaged magnitudes without the amplitude correction suggested by Bono et al. (1995), since the magnitude of the equivalent static star, according to the latter's models, are always within 0.02 mag of the intensitymean value (see Marconi et al. 2003). For the average colors, in turn, we have used the Cacciari et al. static colors, which include the Bono et al. amplitude-dependent corrections. Accordingly, the total number of RR Lyrae stars in our study is 133, including 67 fundamental-mode (RRab or RR0) pulsators with regular light curves and 43 presenting the Blazhko effect $\left(R_{\text {bko }}\right)$, plus 23 pulsating in the first overtone (RRc or RR1 stars).

For nonvariable stars, in turn, we used the extensive photometric database by Ferraro et al. (1997), which includes $B V I$ data for around 45,000 stars. For stars in the outer regions of M $3(r>2$ ), they used both CCD data obtained at the $3.6 \mathrm{~m}$ CFHT telescope and data obtained using photographic plates (Buonanno et al. 1994), whereas for the inner regions Hubble Space Telescope (HST) photometry obtained with WFPC2 was provided. In this work we have adopted their $B V$ and $V I$ data for M3's outermost and innermost regions, respectively. As discussed in the Appendix, there may be a problem with the calibration of their $B$-band (photographic) data, so that we have effectively utilized only their VI data to infer mass values for individual stars.

\subsection{Evolutionary tracks}

We used the set of (canonical) HB evolutionary tracks from Catelan et al. (1998a) for a chemical composition $Y_{\mathrm{MS}}=0.23$ (envelope helium abundance on the zero-age main sequence), $Z=0.001$. These are the same evolutionary models that were used in Catelan (2004), and they also form the basis for the recent calibration of the RR Lyrae period-luminosity relation by Catelan et al. (2004). As a result, we have a total of 22 evolutionary tracks for masses between 0.496 and $0.820 M_{\odot}$, extending from the zero-age HB (ZAHB) to core helium exhaustion.

The possibility of a spread in helium abundances, as recently suggested by several different authors in the case of very massive/peculiar clusters, such as NGC 2808, NGC 5139 ( $\omega$ Centauri), NGC 6388, and NGC 6441 (e.g., Caloi \& D'Antona 2007; Piotto et al. 2007), has not been taken into account in this study, due to the lack of empirical evidence supporting such a hypothesis in the specific case of M 3; in particular, all the clusters for which a spread in $Y_{\mathrm{MS}}$ has been suggested present very long and well-populated blue HB "tails," whereas such a feature is lacking in the case of $\mathrm{M} 3^{1}$. In the present paper, we shall accordingly restrict ourselves to the canonical scenario.

\section{The method}

The aforementioned evolutionary tracks are initially provided in the theoretical plane $\left[\log \left(L / L_{\odot}\right), \log \left(T_{\text {eff }}\right)\right]$, whereas the empirical data are in the form of broadband filter-based magnitudes and colors. Therefore, in order to be able to infer the masses of $\mathrm{HB}$ stars on the basis of their positions in a CMD, we have transformed the evolutionary tracks to the observational coordinates $\left(M_{B}, M_{V}, M_{I}\right)$ on the basis of the color transformations and bolometric corrections by VandenBerg \& Clem (2003) for a metallicity $[\mathrm{Fe} / \mathrm{H}] \simeq-1.5$ and abundance of the alpha elements

\footnotetext{
1 After this paper had been submitted, a preprint was published suggesting that a (relatively small) helium excess may be present among the blue HB stars in M3 (Caloi \& D'Antona 2008). Although not explicitly noted by those authors, the color distribution along the HB is well known to be degenerate in terms of second parameter candidates (e.g., Rood 1973), and so "vertical" information must be added in order to properly constrain the problem (e.g., Crocker et al. 1988; Catelan et al. 1998b; Catelan 2005). Accordingly, in a forthcoming study we will extensively apply such tests, using both spectroscopy and photometric diagnostics, to quantitatively constrain the extent to which helium may be enhanced among the cooler blue HB stars of M3 (Catelan et al., in preparation).
} 
$[\alpha / \mathrm{Fe}] \simeq+0.3$ (e.g., Sneden et al. 2004). Interpolation using their tables was carried out with the algorithm by Hill (1982).

In order to increase the internal precision in the mass determination, we have generated additional evolutionary tracks, with a separation of $2 \times 10^{-4} M_{\odot}$ between consecutive values, by interpolating (also using the Hill 1982 algorithm) along the original tracks available to us. Note that Hill's is a very powerful Hermite interpolation algorithm, which indeed proved of great assistance in dealing with the non-linearities that are observed in the shapes of the evolutionary tracks as a function of mass.

Apparent magnitudes of M3 HB stars have been transformed to absolute magnitudes using a distance modulus $\mu_{V}=$ $15.00 \mathrm{mag}$ in the $V$ band. This is based on a comparison between our evolutionary tracks for $Z=0.001$ and the M 3 observations: as is well known, HB stars spend most of their lifetimes close to the ZAHB, and this is only consistent with our theoretical models for a distance modulus close to the indicated value. On the other hand, Harris (1996) provides $\mu_{V}=15.12$ mag instead; if this value were adopted, we would find the rather untenable result that more than $80 \%$ of M3's HB stars would be in a very advanced evolutionary stage. Below, we discuss the effect of uncertainties in $\mu_{V}$ upon our results.

The colors were corrected for reddening using values for $E(B-V)$ and $E(V-I)$ of 0.010 (Harris 1996) and $0.016 \mathrm{mag}$ (Rieke \& Lebofsky 1985), respectively. The adopted $E(B-V)$ value is only $0.003 \mathrm{mag}$ smaller than the one implied by the Schlegel et al. (1998) maps.

We inferred the mass and the evolutionary times (given as number fractions, where $0 \%$ corresponds to the ZAHB and $100 \%$ to the helium exhaustion line ["terminal age HB," or TAHB]) for each individual HB star by choosing the track that most closely matched the star's observed CMD position (Figs. 1 and 2), with individual error bars being based on the observational (photometric) errors (see Fig. 3). We have also carried out tests in which the evolutionary times are explicitly taken into account in the mass derivation (Sect. 3.1.3), but we have not found important differences in the inferred mass distribution, at least in the case of M3-like HB morphologies, relative to this more straightforward procedure.

For the cases in which no individual error bars are provided in the original photometry, we have computed synthetic observational error bars using the method described by Catelan et al. (2001b, see their Sect. 3.1), which invokes an exponential law for the errors in the observed magnitudes. The photometric error bars are of particular importance in the cases of stars lying close to the dashed line in Fig. 1, since they define whether a star can reasonably be assigned to the HB phase or not (see also Fig. 3, right panel).

In this sense, for stars falling at positions above the TAHB or below the ZAHB (dotted lines in Fig. 1), we used these observational errors to determine, where applicable, the most likely mass value allowed for by the latter. We considered that magnitude and color errors ( $\sigma_{M_{V}}$ and $\sigma_{\text {color }}$, respectively) are standard deviations of a normal distribution centered at the most probable value, which implies that a star has the same probability of having a mass value determined by the points located at $\left[M_{V} \pm \sigma_{M_{V}},(B-V)_{0}\right]$ or $\left[M_{V},(B-V)_{0} \pm \sigma_{\text {color }}\right]$. In fact, one has concentric "isoprobability" ellipses centered at $\left[M_{V},(B-V)_{0}\right]$ (see Fig. 3). The mass assigned to a star thus corresponds to the track in our grid of evolutionary tracks intersecting the error ellipse with the smallest semi-major axis. We used the ellipse with semi-major axis equal to the observational errors to assign errors to individual mass values $\left(\sigma_{M}\right)$. Note, however, that some stars can fall much above the TAHB or below the ZAHB limits,

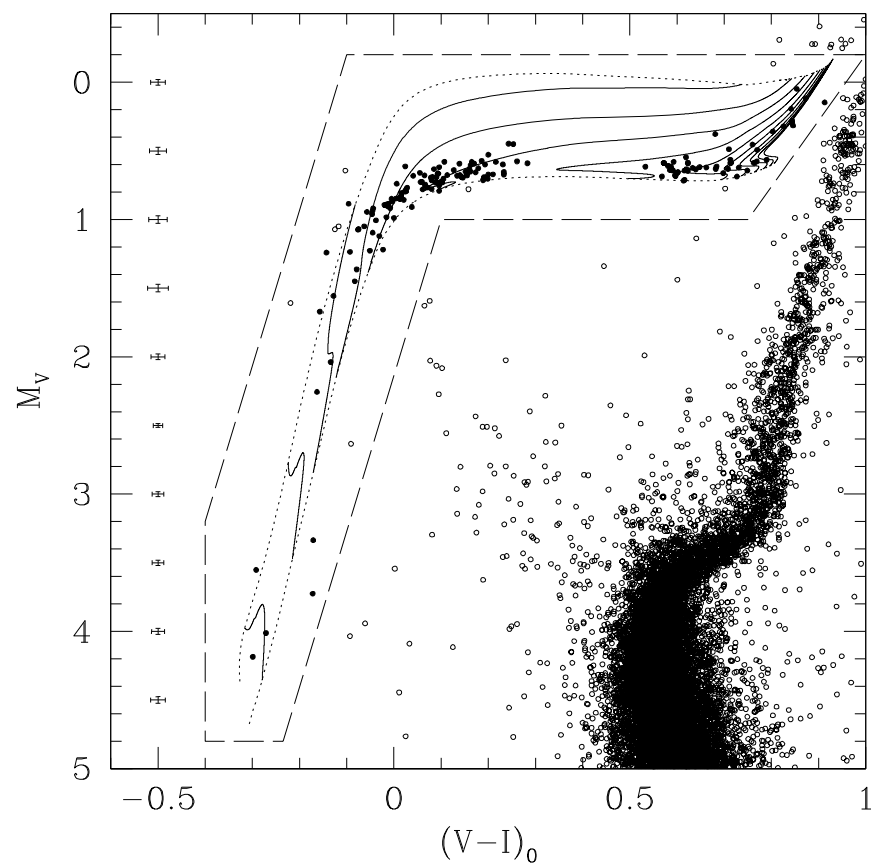

Fig. 1. Evolutionary tracks for the adopted chemical composition (solid lines), along with the corresponding ZAHB and helium exhaustion loci (dotted lines), are overplotted on the CMD for nonvariable stars (open circles) in the inner region of M3. The evolutionary tracks correspond to masses from $0.5 M_{\odot}$ (left) to $0.8 M_{\odot}$ (right), increasing in intervals of $0.025 M_{\odot}$. The locus occupied by HB stars is schematically indicated by the dashed lines; stars within this region plotted with solid circles have had mass values inferred using our procedure. As can be seen, the adopted distance modulus, $\mu_{V}=15.00 \mathrm{mag}$, leads to a close agreement between the observed and predicted lower envelopes of the HB distribution in this diagram.

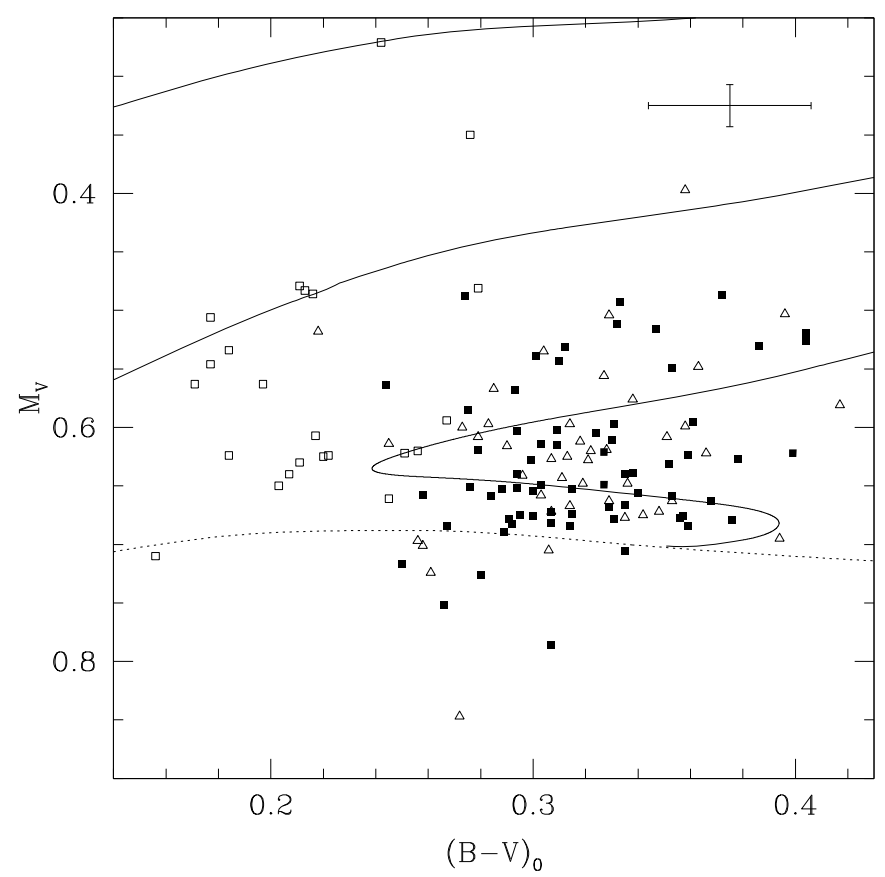

Fig. 2. Evolutionary tracks for the adopted chemical composition (solid lines), along with the corresponding ZAHB (dotted line), are overplotted on the CMD for M3 RR Lyrae variable stars. Different symbols are used for the RRab (filled squares), RRc (empty squares), and $\mathrm{RR}_{\mathrm{bko}}$ (triangles) stars. The solid lines, from left to right, represent evolutionary tracks for $0.600,0.625$, and $0.650 M_{\odot}$. A distance modulus $\mu_{V}=15.00$ mag was again adopted. 

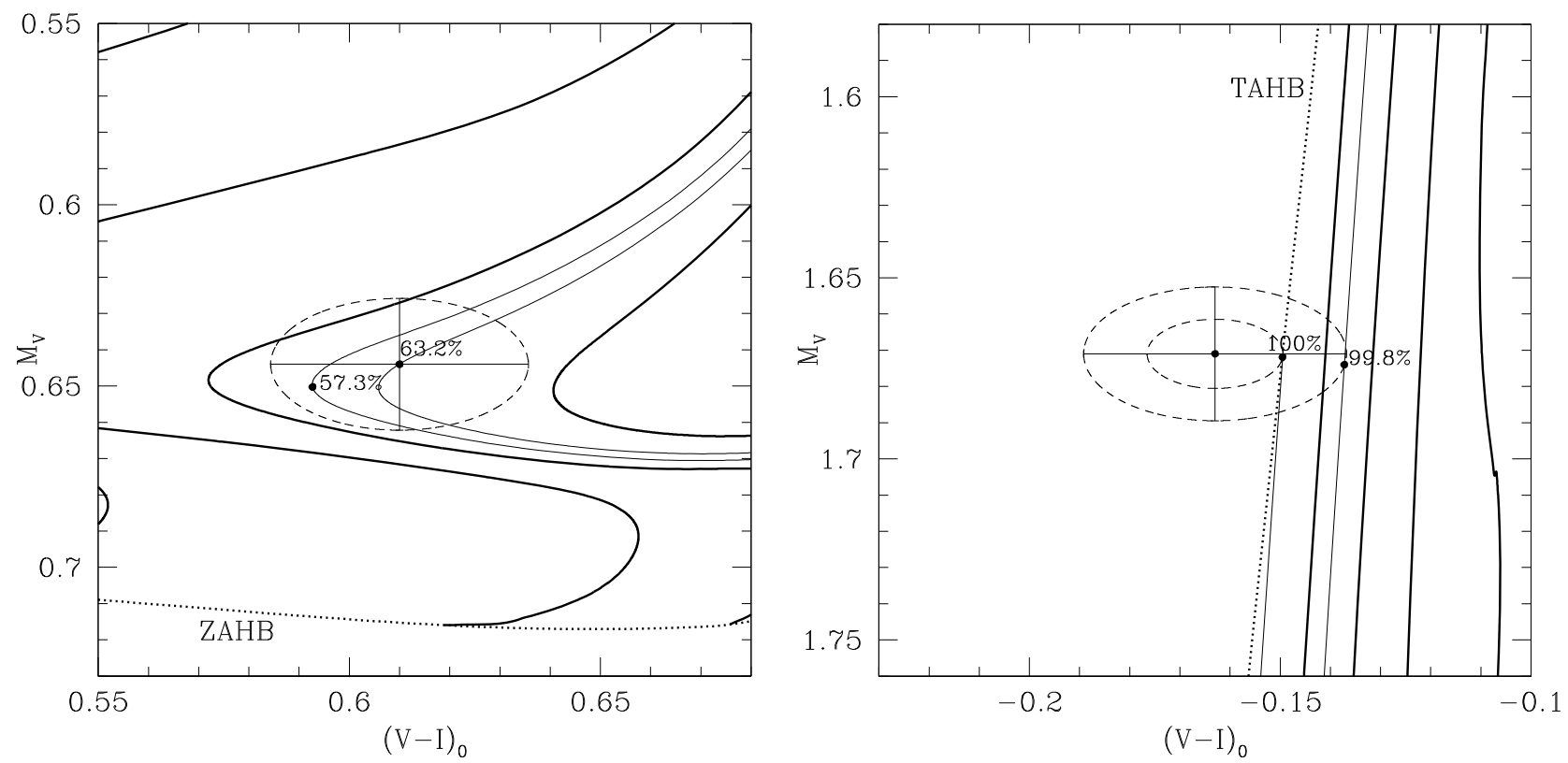

Fig. 3. Method used to determine the mass for each data point. Left panel: a red HB star. The ZAHB is shown as a dotted line, whereas bold lines show evolutionary tracks for $0.65,0.66,0.67$ and $0.68 M_{\odot}$ (from left to right). The ellipse is the isoprobability contour for the 1 -sigma error bars. The mass corresponding to the evolutionary track that passes closest to the center of the ellipse (thin solid line, right) is $0.6744 M_{\odot}$, whereas that corresponding to the minimum evolutionary speed (thin solid line, left) is $0.6726 M_{\odot}$. The evolutionary lifetime $t_{\mathrm{evol}}$ along the HB is also shown in both cases, given as a percentage of the total HB lifetime. Right panel: a blue HB star. Bold lines are evolutionary tracks for 0.545, 0.550, 0.555 and $0.560 M_{\odot}$ (from left to right). Here no HB track goes through the actual data point, and so we adopt for the star the mass corresponding to the track that comes closest to it, and still inside its 1-sigma error ellipse - in this case, $0.541 M_{\odot}$, which corresponds to a star at He exhaustion (thin solid line, left). The evolutionary track for the mass value with the smallest evolutionary speed that still goes through the 1-sigma ellipse is shown as a thin solid line (right); this corresponds to a star with a mass of $0.547 M_{\odot}$ that has completed $99.8 \%$ of its HB evolution.

implying that they are not bona-fide HB stars within their assigned photometric errors (i.e., at the $2-\sigma$ level). For these stars, whose exact number depends on the distance modulus adopted (see Sect. 3.1.2 below), we do not attempt to assign individual mass values, since these would not be physically meaningful.

\subsection{Reliability tests}

In order to ascertain the reliability of our adopted procedure, we have generated synthetic CMDs for the M3 HB, including synthetic photometric errors, to verify whether our method is able to successfully recover a bimodal mass distribution, if one is present, given the uncertainties in the photometry and in the distance modulus. Since for the synthetic models the mass distribution is known a priori, this provides us with a crucial test of whether the mass distribution to be inferred from the actual observations can be trusted, insofar as the presence (or lack) of bimodality is concerned.

In a blind experiment, one of us (M.C.) computed the synthetic distribution and shifted the derived distributions using a distance modulus/reddening combination not known to the other (A.V.) - who in turn attempted to infer the corresponding mass distribution (see below). Reassuringly, the distance modulus and reddening favored by A.V. in the process were in excellent agreement (i.e., to within $0.01 \mathrm{mag}$ in both magnitude and color) with the input values, which in turn were very similar to those given in the Harris (1996) catalog, namely: $\mu_{V}=15.1, E(B-V)=0.01$.

\subsubsection{Synthetic distributions}

We have constructed four input bimodal distributions using the Monte Carlo code SINTDELPHI (Catelan 2004, and references therein), as shown in the left panels of Fig. 4. These mass distributions are closely patterned after the Castellani et al. (2005) proposed bimodal mass distribution for M 3 (see Sect. 1). Figure 4 (right panels) shows the recovered mass distributions, on the basis of our method, assuming the same distance modulus as used when constructing the synthetic distributions (i.e., $\left.\mu_{V}=15.1\right)$. While it is clear that the added synthetic errors necessarily lead to some loss of information, and therefore to somewhat wider (inferred) mass distributions than in the synthetic models, our method always succeeds in recovering a bimodal distribution. Indeed, according to the KMM test (Ashman et al. 1994), the distributions shown on the right-hand panels of Fig. 4 are better described by bimodal rather than unimodal Gaussian distributions, with a probability always higher than $99.99 \%$.

\subsubsection{Synthetic distribution with variable distance modulus}

While the above results are suggestive, as we have seen there appears to be some uncertainty in the M 3 distance modulus, which may also impact the derived mass distribution. We have investigated this source of systematic error by changing the adopted distance modulus over a wide range, from $15.0 \mathrm{mag}$ to $15.4 \mathrm{mag}$, which should cover the full range of acceptable values (we recall that the value used in the simulations is $15.1 \mathrm{mag}$ ). In Fig. 5, we show our results for three different input synthetic bimodal distributions (upper panels) for different values of the adopted distance modulus, from $15.0 \mathrm{mag}$ (second row) to $15.4 \mathrm{mag}$ (bottom row). While the choice of distance modulus does seem to affect the retrieved location of the peaks of the two output mass Gaussians, the bimodality in the mass distribution is always successfully recovered. Additional calculations and statistical tests using the KMM statistic confirm that we only (mistakenly) 


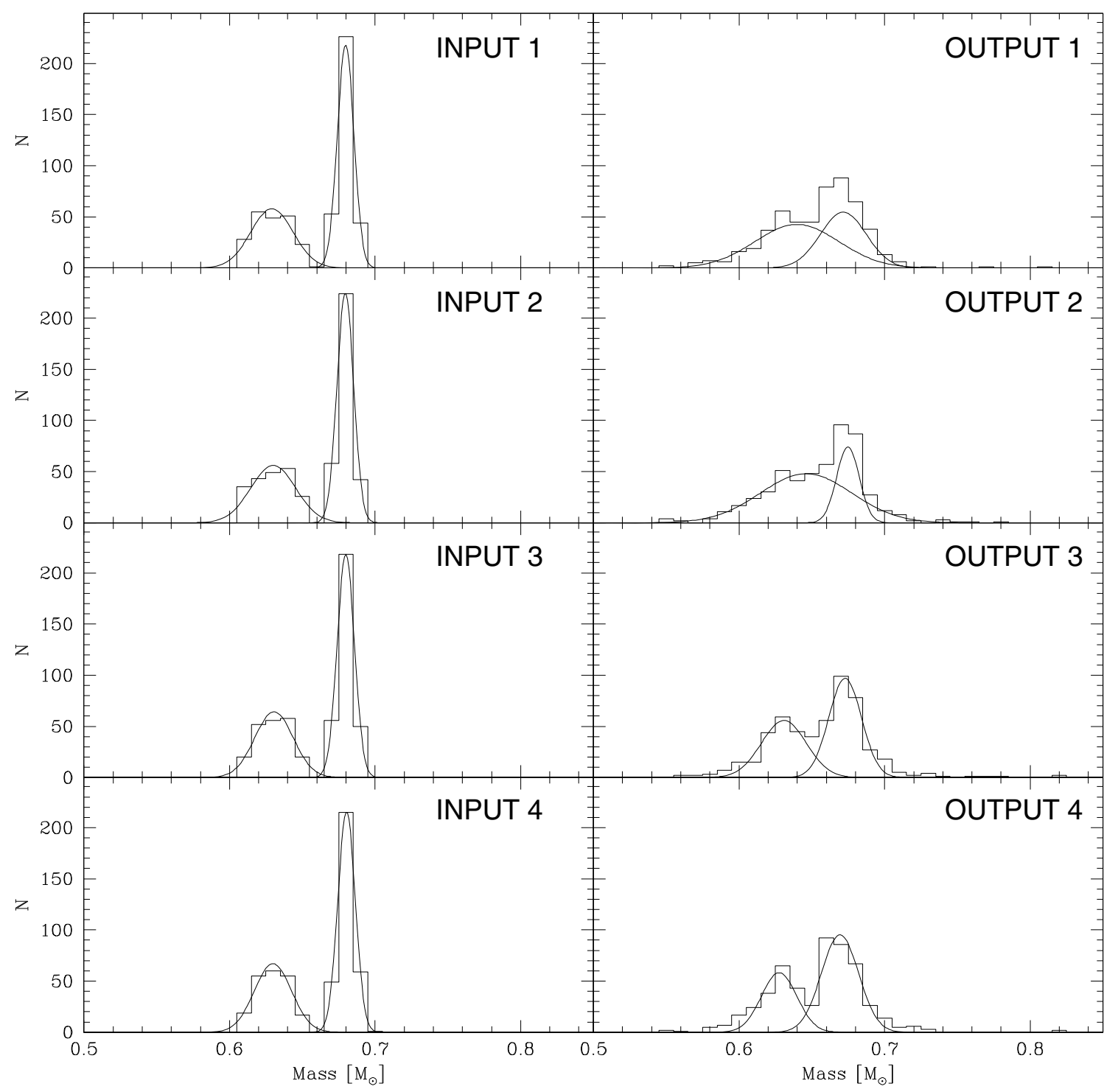

Fig. 4. Bimodality test: for four input bimodal mass distributions (left panels) we determined the mass distribution using our method (right panels). Due to the observational errors, we could not recover precisely the same input distributions, but in no case has a unimodal mass distribution been derived on the basis of an input bimodal distribution.

retrieve a unimodal mass distribution when the adopted distance modulus is in error by more than about 0.3 mag. Note that, when a distance modulus value that is less than the correct one by over $0.1 \mathrm{mag}$ is assumed, we are unable to assign mass values to more than about $50 \%$ of the stars, because many will then fall below the ZAHB (see the last paragraph preceding Sect. 3.1). Naturally, such a loss of stars serves as an indicator that we have an incorrect distance modulus. Thus, our method seems to be very robust in its ability to detect mass bimodality among HB stars in globular clusters.

\subsubsection{Two methods for the determination of the mass distribution}

While the method we used to infer masses looks for the evolutionary track that passes closest to the actual data point in the CMD, other methods can be devised in which the evolutionary times are used as a diagnostic criterion. In particular, to avoid an excess of stars close to the helium exhaustion line, where evolution is very fast, one can alternatively choose the evolutionary track that goes through the 1-sigma error elipse with the smallest evolutionary speed, thus in practice giving higher weight to slower evolutionary stages. How would these different criteria affect the inferred mass distribution?

In order to properly determine the evolutionary speed $v_{\text {evol }}$, we first have to transform the CMD into a plane in which color and magnitude have comparable weights. This has been achieved using a technique similar to that described in Dixon et al. (1996), Catelan et al. (1998a), and Piotto et al. (1999), but here adapted to the $M_{V}, V-I$ plane. Accordingly, we define rescaled "color" $c$ and "brightness" $b$ coordinates as follows:

$c=168.3(V-I)_{0}+96.64$,

$b=-42.67 M_{V}+281.6$.

With this definition, $v_{\text {evol }}$ is computed along an evolutionary track as

$v_{\mathrm{evol}}=\frac{\sqrt{\Delta c^{2}+\Delta b^{2}}}{\Delta t}$.

Having thus defined the evolutionary speed, we have carried out numerical tests in which we generated, using SINTDELPHI, a 


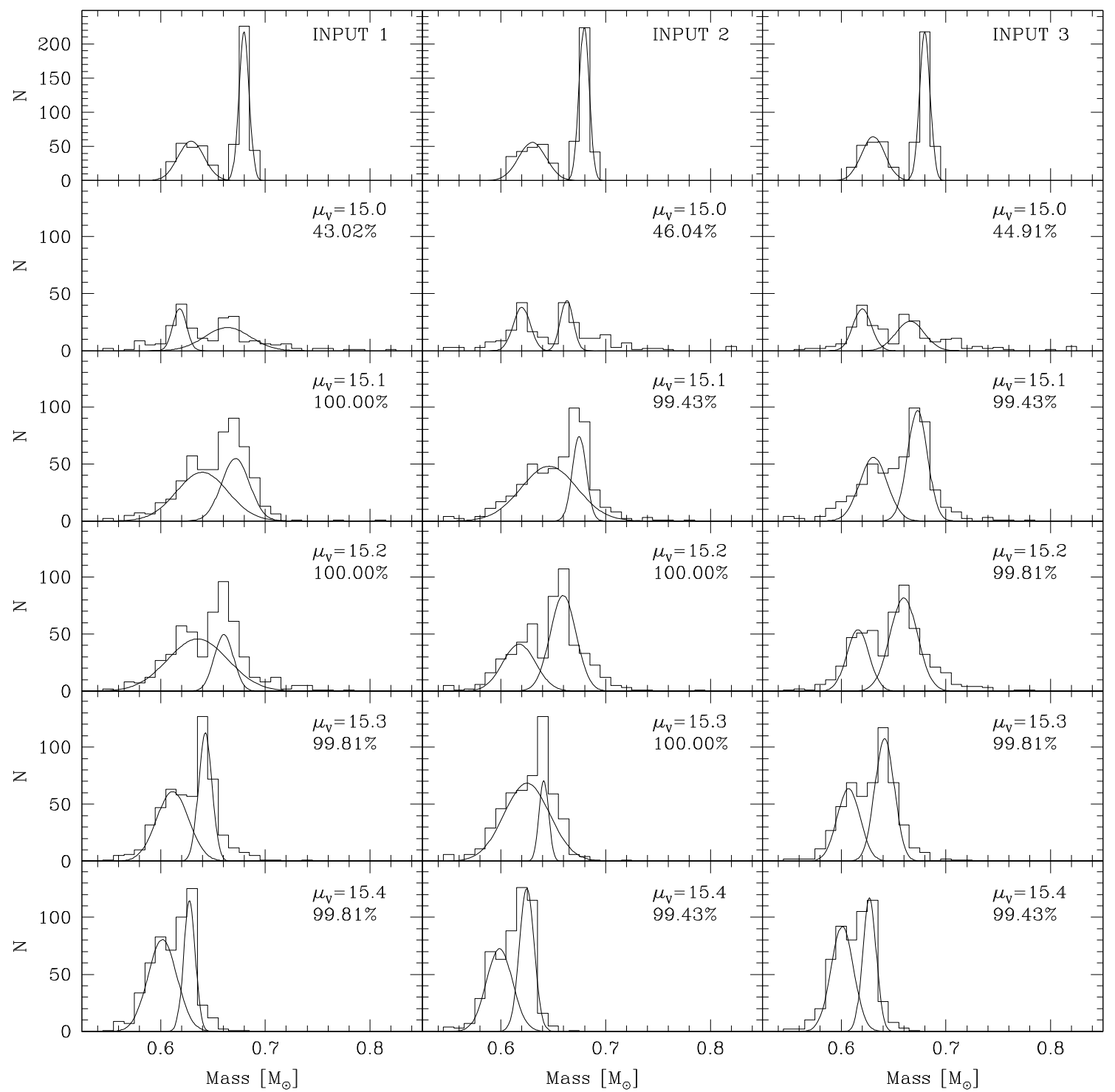

Fig. 5. Distance modulus test: for three input bimodal mass distributions (upper panels) and assuming different distance moduli (as indicated in the insets, along with the percentage of recovered HB stars), we have derived the mass distribution using our method. Input distributions 1 to 3 are the same as in the previous figure. As can clearly be seen, we are always able to recover the input bimodality, albeit not without incurring some systematic errors in the placement of the mass peaks.

synthetic CMD with 1500 synthetic stars and an input mass distribution characterized by $\langle M\rangle=0.642 M_{\odot}, \sigma_{M}=0.020 M_{\odot}$. Synthetic error bars were added as in Catelan et al. (2001b). We then checked the impact of the different criteria upon the inferred mass distribution.

In Fig. 6 we compare the mass distributions that were inferred using the two different criteria indicated in the beginning of this section with the input distribution. In the middle upper panel, the evolutionary track passing closest to the actual data point was selected. In the middle bottom panel, the evolutionary track with the lowest evolutionary speed $v_{\text {evol }}$ within the 1sigma error ellipse was in turn selected. The differences between the mass distributions derived using either of these two methods are very small, and they are both clearly able to properly reproduce the input mass distribution. On the other hand, the method does not perform as accurately for more extreme HB types. This is also revealed by Fig. 6, where we show our attempts to recover the input mass distribution in the cases of simulations computed for extremely blue (left panel) and red (right panel)
HB morphologies. Irrespective of whether we adopt the purely geometrical criterion or the criterion involving the minimum $v_{\text {evol }}$ (upper and lower panels, respectively), we are unable to reliably recover the input mass distribution in such cases. Photometry in other, more suitable bandpasses, where one finds a stronger dependence of colors and magnitudes on the stellar mass, would be required to achieve better results when the HB distribution is comprised of very blue or very red stars.

We thus conclude that the simple method (in which the evolutionary track passing closest to the data point in the CMD is selected) appears to be good enough for our purposes, since it performs quite well for rather even, M 3-like HB morphologies.

\section{Results}

\subsection{Mass distribution along the $\mathrm{HB}$ of $\mathrm{M} 3$}

The mass distribution of stars along the HB of $\mathrm{M} 3$ was obtained separately for the nonvariable and variable stars, since we had to rely on empirical data in the VI bands for the former and in the 


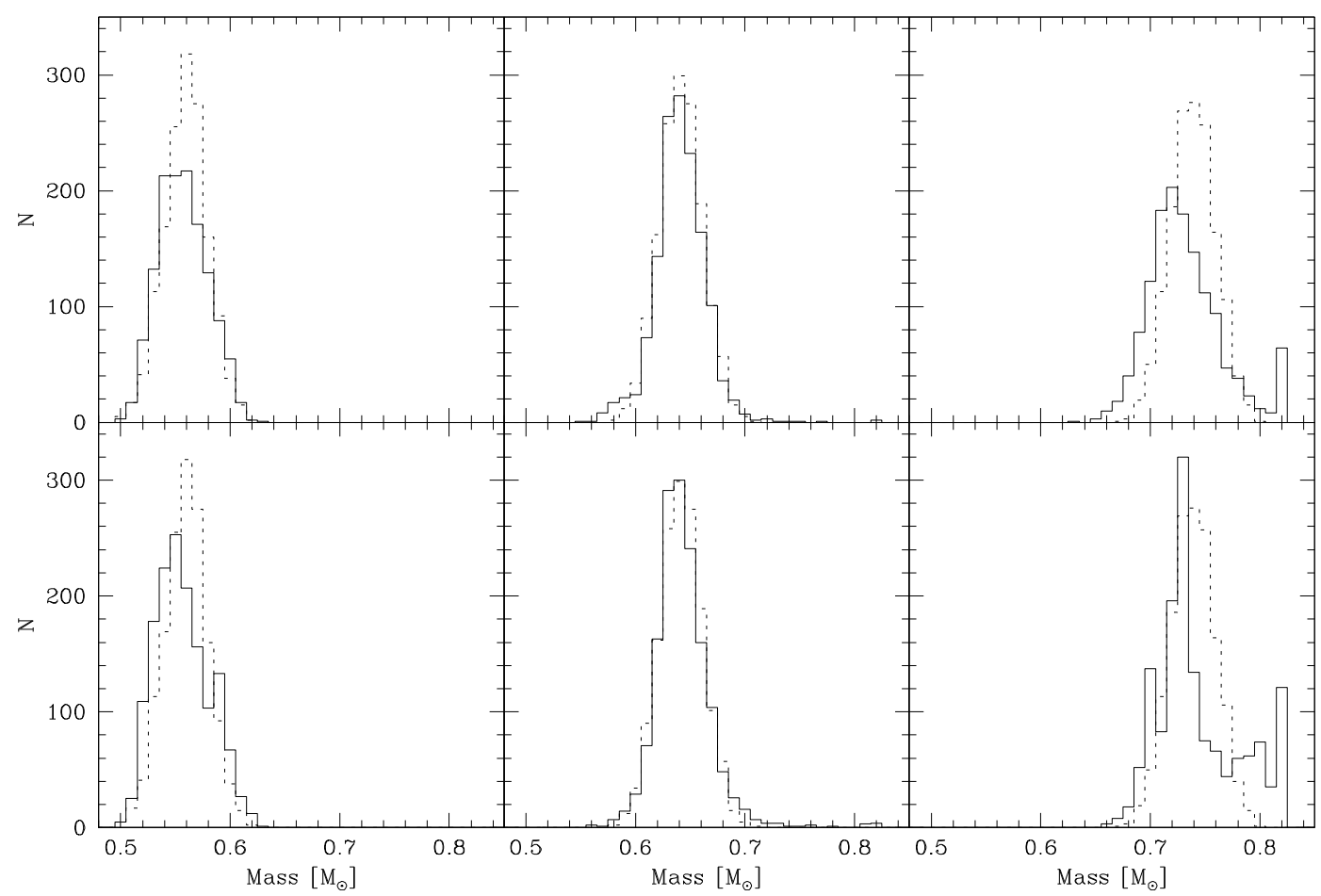

Fig. 6. Methodology test: for known input mass distributions (dashed lines) HB simulations with 1500 stars were computed as described in the text, and the mass distribution was then inferred (solid lines) from the resulting CMD distribution using two different methods: evolutionary tracks that pass closest to the individual data points (upper panels) and evolutionary tracks that pass through the 1- $\sigma$ error ellipse with the smallest evolutionary speed $v_{\text {evol }}$ (bottom panels). On the left and right panels the cases of completely blue and completely red HB morphologies are shown, respectively, whereas the middle panel refers to the case of a more even distribution along the CMD, as more appropriate in the case of M 3 . While the present method becomes less reliable for extreme HB types, it does provide an excellent description of the input mass distribution for an M3-like HB morphology.

$B V$ bands for the latter. Our final M 3 mass distribution corresponds to the sum of these two, separately derived, distributions.

The non-variable stars that we selected for our study are those pertaining to the inner regions of M 3. We inferred masses for 157 stars falling sufficiently close to HB evolutionary tracks, including 105 stars on the blue $\mathrm{HB}$ and 52 on the red $\mathrm{HB}$ (filled circles in Fig. 1). The stars plotted with open circles within the dashed region of Fig. 1 are those for which mass values were not assigned, since their photometry places them several $\sigma$ away from the predicted HB locus. The resulting mass distribution for these non-variable stars is bimodal, with peaks centered at $0.625 M_{\odot}$ and $0.665 M_{\odot}$ (Fig. 7). The two peaks reflect the masses of blue and red HB stars, respectively; naturally, we anticipate that the gap in between these two mass modes will be at least partially filled when due account is taken of M3's variable stars. Had we used the method described by Rood \& Crocker (1989), we would not have found any non-variable stars with masses around $0.640 M_{\odot}$, since in that method the effects of evolution away from the ZAHB are not taken into account.

The mass distribution for variable stars (Fig. 8, upper panel) was obtained from 127 of the 133 stars that we chose initially. The remainder of the stars fall below the ZAHB, and their photometric errors do not allow them to be reconciled with the HB phase (some of them may be pre-ZAHB stars). A Gaussian fit provides a mean mass of $0.6445 M_{\odot}$ and dispersion $0.0076 M_{\odot}$. More in detail, a Gaussian fit to the mass distribution for the RRab stars has a mean mass and dispersion given by $0.6440 M_{\odot}$ and $0.0049 M_{\odot}$, respectively (Fig. 8, second panel), whereas the Blazhko-type RR Lyrae have mean mass $0.6482 M_{\odot}$ and dispersion $0.0085 M_{\odot}$ (Fig. 8, bottom panel). For the RRc, in turn, a rather uniform mass distribution is inferred instead,

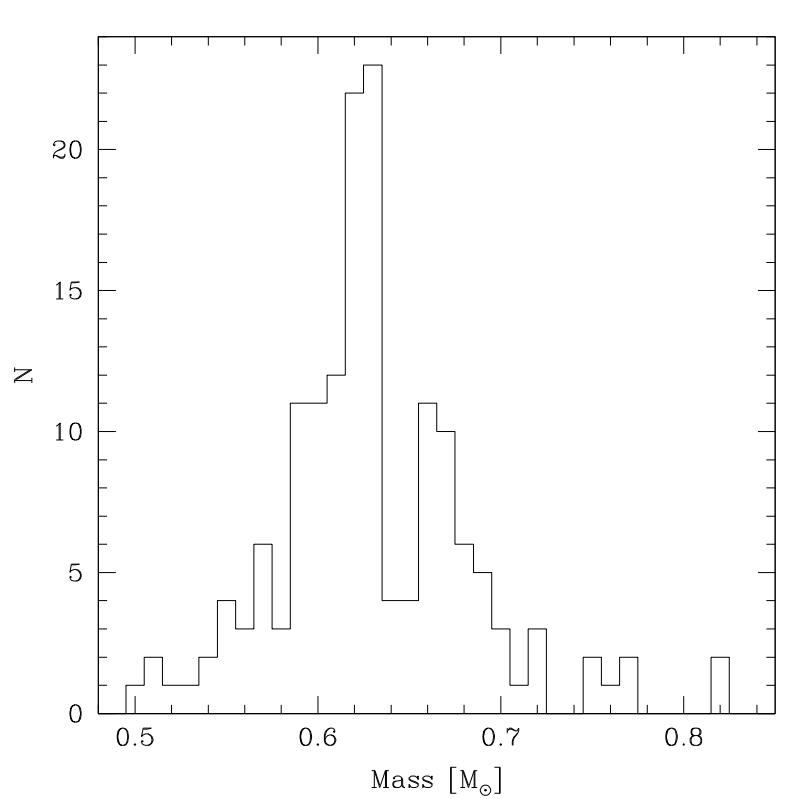

Fig. 7. Mass distribution of nonvariable stars in the inner regions of M3. It displays two maxima, produced by stars in the red and blue HB domains. The non-zero minimum in between indicates the existence of stars in highly evolved stages towards the end of the HB phase.

though in this case the number of stars is much lower (Fig. 8, third panel). Note that the derived masses for the RRc stars tend to be lower than for the RRab stars, which is consistent with the expectations of canonical theory (see, e.g., Fig. 2). 


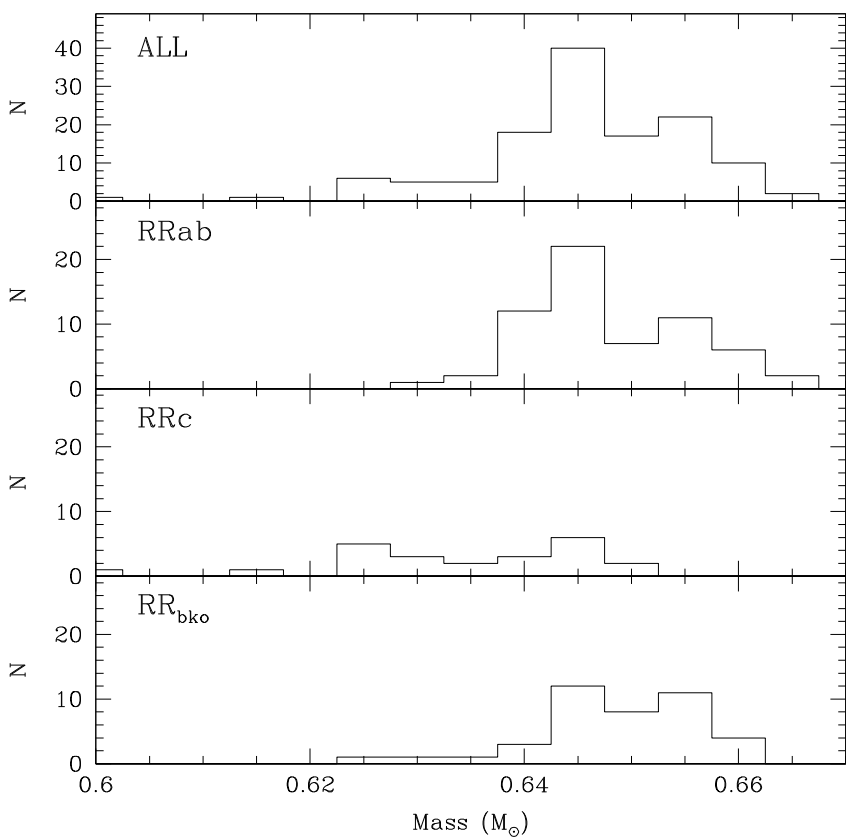

Fig. 8. Upper panel: inferred mass distribution for all RR Lyrae variable stars in M3. The mass distributions for each RR Lyrae subtype are shown in the other panels: RRab with well-behaved light curves (second panel), RRc (third panel), and RR Lyrae showing the Blazhko effect (bottom panel).

In order to study the global mass distribution along the M3 HB, we must suitably combine our samples of variable and non-variable stars. In particular, we must ensure consistency with the observed proportions of stars falling along the blue $\mathrm{HB}$, the red $\mathrm{HB}$, and inside the instability strip. We accordingly use the proportions $\mathcal{B}: \mathcal{V}: \mathcal{R}=39: 40: 21$ (Catelan 2004), where $\mathcal{B}, \mathcal{V}$, and $\mathcal{R}$ indicate the numbers of blue, variable, and red HB stars, respectively. As a consequence, given the sample of red HB stars in our study, in order to derive an unbiased, final mass distribution we must randomly remove 9 and 28 stars from our blue HB and RR Lyrae samples, respectively. Our final derived mass distribution is shown in Fig. 9.

We fit a Gaussian to this mass distribution, finding (Fig. 9)

$\langle M\rangle=0.642 M_{\odot}, \quad \sigma=0.020 M_{\odot}$.

This result is consistent with that previously derived by Rood \& Crocker (1989), who also find, using a method similar to ours but not taking into account evolutionary effects, a unimodal mass distribution, with $\langle M\rangle=0.666 M_{\odot}$ and $\sigma=0.018 M_{\odot}$. Catelan et al. (2001b) have also found that a unimodal mass distribution along the M $3 \mathrm{HB}$ is consistent with the observed HB morphology parameters of the cluster: according to their results, the innermost cluster regions may be characterized by $\langle M\rangle=0.637 M_{\odot}$ and $\sigma=0.023 M_{\odot}$, whereas the outermost regions, which seem to be redder, may be described instead in terms of a normal distribution with $\langle M\rangle=0.645 M_{\odot}$ and $\sigma=0.018 M_{\odot}$.

On the other hand, while the fit shown in Fig. 9 seems acceptable, close inspection reveals what appears to be an excess of stars on the wings of the distribution, especially at its lowmass end. That the true mass distribution may be unimodal but not precisely Gaussian is also supported by an analysis of its skewness and kurtosis (see Sect. 14.1 in Press et al. 1992). We find values of Skew $=0.28 \pm 0.16$ and Kurt $=3.31 \pm 0.31$, whereas a perfect Gaussian has Skew $\equiv 0$ and Kurt $\equiv 3$.

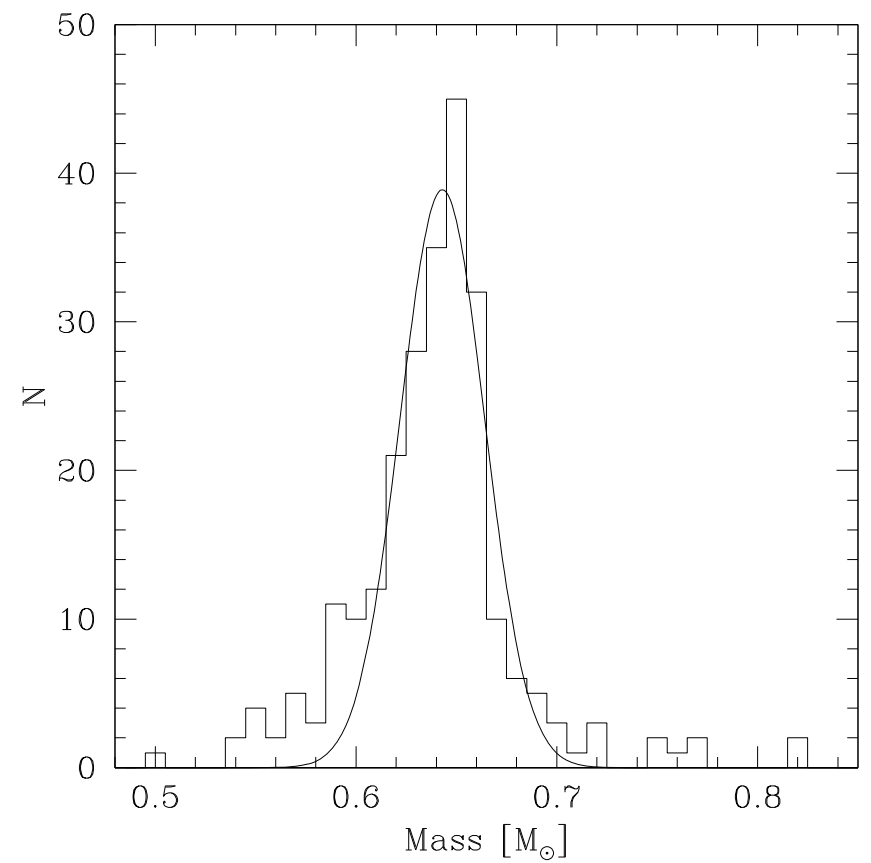

Fig. 9. Mass distribution for the M $3 \mathrm{HB}$, obtained by combining the mass distributions for the variable and non-variable stars. The bestfitting Gaussian (solid line) has $\langle M\rangle=0.642 M_{\odot}$ and $\sigma=0.020 M_{\odot}$.

Naturally, since the derived unimodal distribution is not perfectly Gaussian, an alternative description of the data can be accomplished with a linear combination of Gaussians. Indeed, when faced with the question to opt for a single Gaussian or a combination of two Gaussians, the KMM test gives, not surprisingly, preference for the latter, at a very high level of confidence $(>99.99 \%)$. The best-fitting bimodal solution is shown in Fig. 10, where the dashed line shows the result of the sum over the two derived Gaussians (solid lines). In this case, the bestfitting Gaussians are given by

$$
\begin{array}{ll}
\langle M\rangle_{1}=0.633 M_{\odot}, & \sigma_{1}=0.026 M_{\odot}, \\
\langle M\rangle_{2}=0.650 M_{\odot}, & \sigma_{2}=0.008 M_{\odot},
\end{array}
$$

with $71.1 \%$ of the stars in the first mode and $28.9 \%$ in the second.

As previously discussed on the basis of Figs. 4 and 5, one expects that the mass distribution determined in this way will differ somewhat from the "intrinsic" mass distribution of the cluster. We have made an effort to account for this effect, at least in the case of the bimodal solution, by comparing the derived and input mass modes, as given by the panels with $\mu_{V}=15.1$ in Fig. 5. As a consequence, we infer that the "true" mass modes have on average $\sigma$ values which are only about $77 \%$ of those given above. Similarly, the positions of the centers of the high- and low-mass modes are on average shifted by $-0.008 M_{\odot}$ and $+0.008 M_{\odot}$, respectively. Therefore, the suggested (corrected) mass distribution for the M 3 stars, assuming a bimodal solution, is the following:

$$
\begin{array}{ll}
\langle M\rangle_{1, \text { cor }}=0.625 M_{\odot}, & \sigma_{1, \text { cor }}=0.019 M_{\odot}, \\
\langle M\rangle_{2, \text { cor }}=0.658 M_{\odot}, & \sigma_{2, \text { cor }}=0.006 M_{\odot},
\end{array}
$$

again with about $71 \%$ of the stars in the low-mass mode and $29 \%$ in the high-mass mode.

Note that our derived mass distribution for M3 differs markedly from either the input or the derived solutions shown 


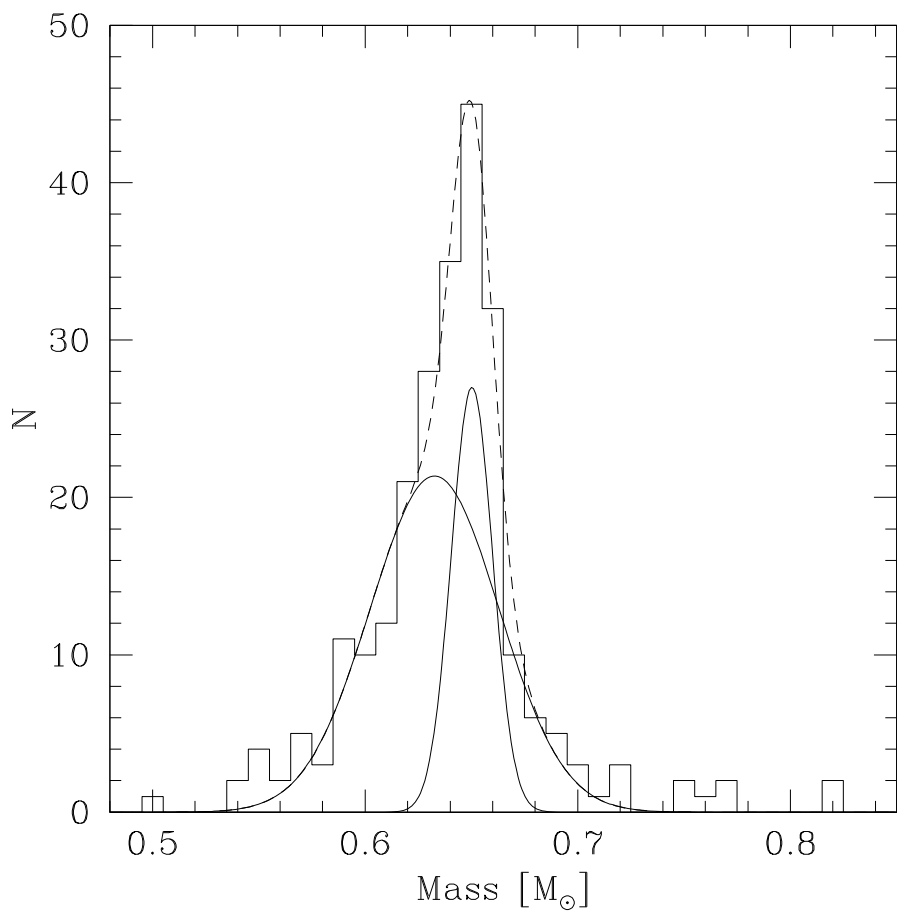

Fig. 10. As in Fig. 9, but showing the best-fitting bimodal solution. The two Gaussians (solid lines) have $\langle M\rangle_{1}=0.633 M_{\odot}, \sigma_{1}=0.026 M_{\odot}$ (with $71.1 \%$ of the stars) and $\langle M\rangle_{2}=0.650 M_{\odot}, \sigma_{2}=0.008 M_{\odot}$ (with $28.9 \%$ of the stars).

in Figs. 4 and 5, since the two Gaussians here are much closer together than was derived in almost all distributions except in those with the largest distance moduli (which differed from the correct solution by 0.2 dex or more in $\mu_{V}$ ). Therefore, while our study may give some support to the existence of a degree of HB bimodality in M3, the derived distribution also differs in detail from the one by Castellani et al. (2005). Interestingly, the two derived mass modes do not seem to be fully detached, contrary to what was suggested in the latter study. As a consequence, synthetic HBs based on the mass distribution that was inferred in this section are again unable to properly reproduce the observed fundamentalized period distribution in M 3 (see Sect. 1 for references to prior work on this topic).

We repeated the whole procedure described in this section, but using the set of tracks independently computed by Pietrinferni et al. (2004). As a result, we derived a mass distribution that is qualitatively very similar to that shown in Figs. 9 and 10 . The only noteworthy difference is that, when using the Pietrinferni et al. tracks, the peak of the high-mass mode is slightly more pronounced than indicated in these plots.

\subsection{Evolutionary times}

While we have previously argued (Sect. 3.1.3) that the inferred mass distribution is fairly robust with regard to the different recipes for using the evolutionary lifetimes to infer the mass values, we have so far not discussed what happens to the resulting distribution of evolutionary lifetimes itself. In principle, assuming a smooth feeding of the HB phase from the RGB tip, one should have an essentially flat distribution between 0 and $100 \%$ of the HB lifetime, except of course for statistical fluctuations. What does the inferred distribution of evolutionary times look like, in the case of M3?
The answer is provided in Fig. 11 (left). In this plot, the upper panel shows the derived $t_{\text {evol }}$ distribution, corresponding to the mass distribution that was inferred by simply adopting the evolutionary tracks that pass closest to each individual data point in the CMD. There appears to be a dearth of stars in the range $t_{\mathrm{evol}} \approx 20-60 \%$, and/or an excess of stars with $t_{\mathrm{evol}} \approx 70-90 \%$. The lower panel in the same figure reveals that, by adopting the smallest $v_{\text {evol }}$ value inside the 1 -sigma error ellipse for each data point, not only is the problem not solved, but also a large excess of stars with $t_{\text {evol }} \approx 10 \%$ results as well, as well as another strong peak of stars with $t_{\mathrm{evol}} \approx 55 \%$. Figure 11 (right) shows the result of the same exercise, performed using the Pietrinferni et al. (2004) evolutionary tracks. The results are qualitatively very similar, thus indicating that the problem does not lie in our specific choice of evolutionary tracks.

The presence of these two peaks is an expected, direct consequence of the minimum $v_{\text {evol }}$ method. As is well known, HB evolution is slowest close to the "turning points" on the CMD, including the blue and red "noses" that are clearly seen in the evolutionary track displayed in Fig. 2. Therefore, any method based on minimum $v_{\text {evol }}$ values will tend to preferentially pick the $t_{\text {evol }}$ values associated with these features - as in the case of Fig. 3 (left panel), where the "blue nose" position was selected for a star whose original position on the CMD implied a slightly more advanced evolutionary stage. We conclude that the peak at $t_{\text {evol }} \approx 55 \%$ can be ascribed to these "blue noses," whereas the peak at $t_{\mathrm{evol}} \approx 10 \%$ is instead due to the "red noses" that occur slightly after the star reaches the ZAHB.

Irrespective of the method adopted, the main problem revealed by Fig. 11 is the fact that there are many fewer HB stars with $0 \% \leq t_{\text {evol }} \leq 50 \%$ than there are stars with $50 \%<t_{\text {evol }} \leq$ $100 \%$. We have checked that this is not a problem affecting only a group of stars along M 3's HB, but the $t_{\text {evol }}$ distribution looks bimodal for red HB, blue HB, and RR Lyrae stars. At present, we do not have an explanation for this problem, other than speculating that the present set of evolutionary tracks predicts too little luminosity evolution, thus leading to too few predicted stars at high luminosities, compared to the observations (see also Sect. 2.2.4 in Catelan et al. 2001a, and Sect. 3.2 in Catelan et al. 2001b). We have checked that this is not a problem exclusively of our adopted models; similar (or even more extreme) discrepancies are suggested by the Pietrinferni et al. (2004) or the Dotter et al. (2007) evolutionary tracks. This is further illustrated in Fig. 12, which shows that, irrespective of the set of models used, HB stars are predicted to spend too little time at relatively high luminosities, compared with what is suggested by the observed CMD. More specifically, along the horizontal part of the $\mathrm{HB}$, one expects to find, according to these models, $\approx 65-70 \%$ of the stars within about $0.05 \mathrm{mag}$ of the ZAHB. The observations, on the other hand, reveal $\approx 40 \%$ of the HB stars within such a magnitude range from the ZAHB, even including in the count those stars that fall below the ZAHB (presumably due to photometric errors; see, e.g., Fig. 2). If we changed the adopted ZAHB position so as to better accomodate these "subZAHB stars," the noted discrepancy would become even more dramatic.

Naturally, until these problems are conclusively solved, mass distributions based on the CMD method, such as the one provided in the present paper, should be considered tentative. Similarly, we caution that the fact that there are also too many (presumably) evolved red HB stars argues against a solution to this specific problem based on a component with a high helium abundance among the blue HB stars. 


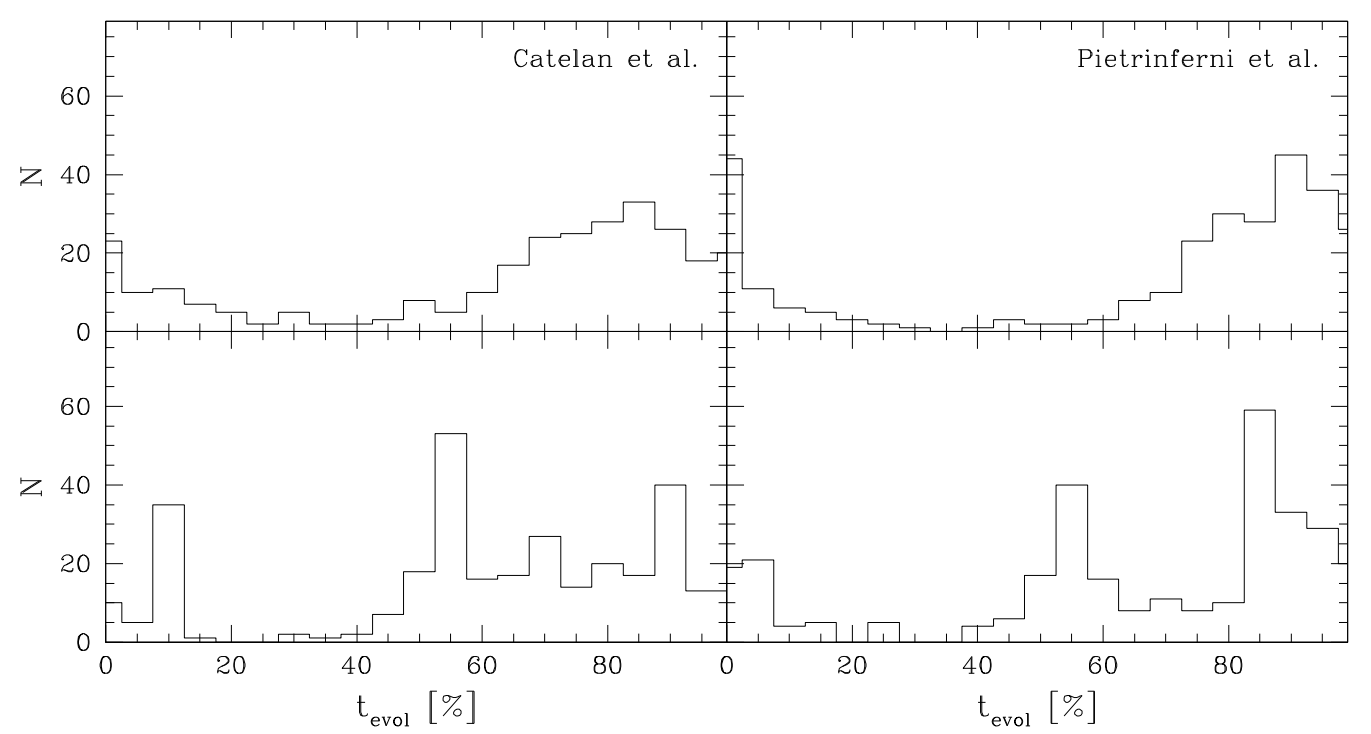

Fig. 11. The derived distributions of evolutionary times $t_{\mathrm{evol}}$ for stars in the HB phase, in fractions of the total HB lifetime for the star's inferred mass (where $0 \%$ corresponds to the ZAHB and $100 \%$ to the TAHB). Left: results obtained on the basis of our own evolutionary tracks; right: results based on the independent set of tracks by Pietrinferni et al. (2004). Upper panels: evolutionary times based on the HB tracks that pass closest to each individual data point in the CMD (e.g., those that would correspond to $t_{\mathrm{evol}}=63.2 \%$ and $100 \%$ for left and right panels in Fig. 3 , respectively). Bottom panels: evolutionary times corresponding to the minimun evolutionary speed inside the 1-sigma error ellipse (e.g., those that would correspond to $t_{\mathrm{evol}}=57.3 \%$ and $99.8 \%$ for the left and right panels in Fig. 3, respectively).

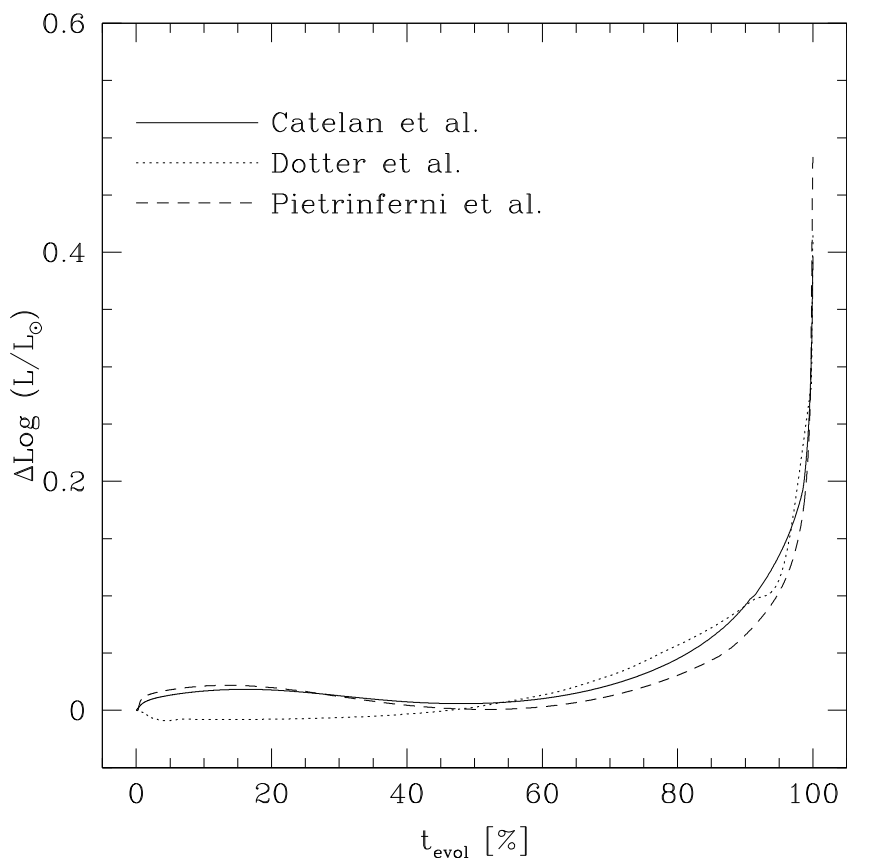

Fig. 12. Luminosity evolution from the ZAHB, $\Delta \log \left(L / L_{\odot}\right) \equiv$ $\log L\left(t_{\text {evol }}\right)-\log L\left(t_{\text {evol }}=0\right)$, for three independent sets of evolutionary tracks. Evolutionary tracks whose ZAHB position lies close to the middle of the instability strip is shown in all cases. According to these models one should expect $\approx 65-70 \%$ of all stars along the horizontal part of the HB to lie within 0.05 mag of the ZAHB. Observations indicate instead that only $\approx 40 \%$ are found within this range (see text).

\section{Summary and conclusions}

In the present paper, we have studied the mass distribution of M3's HB stars by comparing their observed locations in the CMD with the predictions of evolutionary models. Our results suggest that, within the canonical framework, M3's HB mass distribution can be characterized either by a unimodal mass distribution that is not perfectly Gaussian or by a bimodal mass distribution in which the two mass modes are adequately described by Gaussians. In the latter case, the two mass modes appear to be separated in mean mass by only $\approx 0.03 M_{\odot}$, whereas the dispersion in mass of the low-mass mode is significantly higher than that of the high-mass mode.

As far as the distribution of evolutionary times is concerned, we find that it is not in good agreement with the canonical expectations, in that we obtain a bimodal distribution, with a dearth of stars with $t_{\text {evol }} \approx 20-60 \%$ and/or an excess of stars with $t_{\mathrm{evol}} \approx 70-90 \%$. This suggests that the present evolutionary models underestimate the luminosity evolution along the HB. We have checked that other sets of evolutionary tracks, such as the ones computed by Pietrinferni et al. (2004) or Dotter et al. (2007), indicate similar, or even less, luminosity evolution than the present ones. Until this problem is solved and better agreement between observed and predicted $t_{\text {evol }}$ values can be achieved, mass distributions derived using a CMD-based method, such as the one presented in this paper, should be considered tentative. Conversely, no solution for the HB star problems in M 3 that were previously discussed by several authors (Rood \& Crocker 1989; Catelan 2004; Castellani et al. 2005; D'Antona \& Caloi 2008) can be considered complete until good agreement between predicted and observed lifetime distributions is finally achieved (see also Catelan et al. 2001a,b).

Acknowledgements. Support for M.C. is provided by Proyecto Fondecyt Regular No. 1071002. We thank M. Zoccali for interesting discussions, and the anonymous referee for her/his comments which have led to a significantly improved manuscript. Cristián Aruta, in memoriam.

\section{Appendix A: Photometry in the BV bands: is there a problem?}

In Fig. A.1 we overplot our evolutionary tracks with the observational data for the outer regions of M 3. There is a clear disagreement between the predicted locus of M3's blue HB stars and the observations. We find no combination of $\mu_{V}$ and $E(B-V)$ 


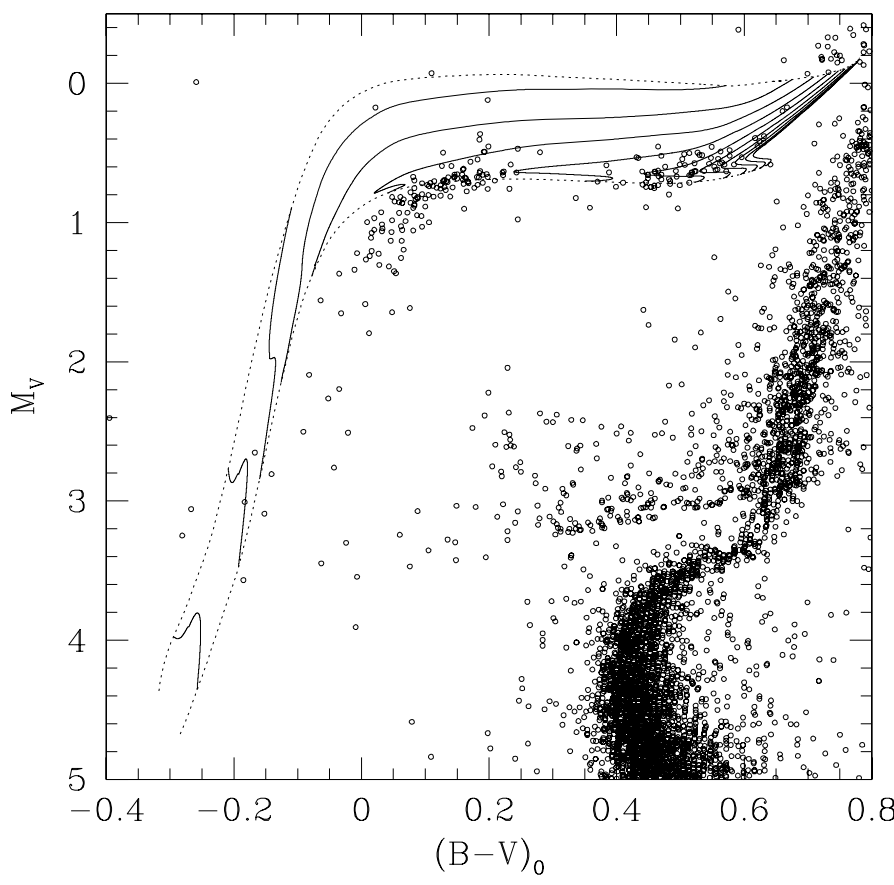

Fig. A.1. $M_{V}, B-V$ CMD of nonvariable stars in the outer regions of M3. Evolutionary tracks (solid lines), along with the corresponding ZAHB and TAHB lines (dotted lines), are overplotted. As can clearly be seen, for colors bluer than $(B-V)_{0}=0.2$ the models progressively deviate from the empirical data. As discussed in the main text, this is likely due to an error in the calibration of the photographic data (compare with Fig. 1, which reveals excellent agreement between the models and the CCD data in the $M_{V},(V-I)_{0}$ plane).

that allows us to provide close agreement between the models and the empirical data. On the other hand, for the inner regions of M3 (Fig. 1) no such problem was present. Since the measurements for the inner regions are based on CCD data (Ferraro et al. 1997) whereas for the outer regions photographic data were used (Buonanno et al. 1994), this strongly suggests an error in the calibration of the photographic data, especially the color term. This is consistent with the discussion in Ferraro et al., who have also called attention to the possibility of large errors in the $B$-band photometry. Note that this problem does not affect the adopted colors and magnitudes for the RR Lyrae stars, whose adopted $B V$ photometry (Sect. 2) came from an independent source (Cacciari et al. 2005, and references therein).

\section{References}

Ashman, K. M., Bird, C. M., \& Zepf, S. E. 1994, AJ, 108, 2348 Bailey, S. I. 1913, Harv. Coll. Observ. Annals, 78, 1 Bono, G., Caputo, F., \& Stellingwerf, R. F. 1995, ApJS, 99, 263 Buonanno, R., Corsi, C. E., Buzzoni, A., et al. 1994, A\&A, 290, 69 Cacciari, C., Corwin, T. M., \& Carney, B. W. 2005, AJ, 129, 267 Caloi, V., \& D'Antona, F. 2007, A\&A, 463, 949

Caloi, V., \& D'Antona, F. 2008, ApJ, in press [arXiv: 0709. 1572]

Caloi, V., Castellani, V., \& Tornambè, A. 1978, A\&AS, 33, 169

Castellani, M., Castellani, V., \& Cassisi, S. 2005, A\&A, 437, 1017

Catelan, M. 2004, ApJ, 600, 409

Catelan, M., Borissova, J., Sweigart, A. V., \& Spassova, N. 1998a, ApJ, 494, 265 Catelan, M., Sweigart, A. V., \& Borissova, J. 1998b, in A Half Century of Stellar Pulsation Interpretation, ed. P. A. Bradley, \& J. A. Guzik (San Francisco: ASP), ASP Conf. Ser., 135, 41

Catelan, M., Bellazzini, M., Landsman, W. B., et al. 2001a, AJ, 122, 3171

Catelan, M., Ferraro, F. R., \& Rood, R. T. 2001b, ApJ, 560, 970

Catelan, M., Pritzl, B. J., \& Smith, H. A. 2004, ApJS, 154, 633

Clementini, G., Corwin, T. M., Carney, B. W., \& Sumerel, A. N. 2004, AJ, 127, 938

Corwin, T. M., \& Carney, B. W. 2001, AJ, 122, 3183

Crocker, D. A., Rood, R. T., \& O'Connell, R. W. 1988, ApJ, 332, 236

Dixon, W. V. D., Davidsen, A. F., Dorman, B., \& Ferguson, H. C. 1996, AJ, 111, 1936

Dotter, A., Chaboyer, B., Jevremović, D., et al. 2007, AJ, 134, 376

Ferraro, F. R., Carretta, E., Corsi, C. E., et al. 1997, A\&A, 320, 757

Harris, W. E. 1996, AJ, 112, 1487

Hill, G. 1982, Publ. Dom. Astrophys. Obs., 16, 67

Kaluzny, J., Hilditch, R. W., Clement, C., \& Rucinski, S. M. 1998, MNRAS, 296, 347

Lee, Y.-W., Demarque, P., \& Zinn, R. 1990, ApJ, 350, 155

Marconi, M., Caputo, F., Di Criscienzo, M., \& Castellani, M. 2003, ApJ, 596, 299

Oosterhoff, P. Th. 1939, Observatory, 62, 104

Oosterhoff, P. Th. 1944, Bull. Astron. Inst. Neth., 10, 55

Pietrinferni, A., Cassisi, S., Salaris, M., \& Castelli, F. 2004, ApJ, 612, 168

Piotto, G., Zoccali, M., King, I. R., et al. 1999, AJ, 118, 1727

Piotto, G., Bedin, L. R., Anderson, J., et al. 2007, ApJ, 661, L53

Press, W. H., Teukolsky, S. A., Vetterling, W. T., \& Flannery, B. P. 1992,

Numerical Recipes in C (Cambridge: Cambridge Univ. Press)

Rieke, G. H., \& Lebofsky, M. J. 1985, ApJ, 288, 618

Roberts, M. S., \& Sandage, A. 1995, AJ, 60, 185

Rood, R. T. 1973, ApJ, 184, 815

Rood, R. T., \& Croker, D. A. 1989, in The Use of Pulsating Stars in Fundamental Problems of Astronomy, ed. E. G. Schmidt (Cambridge: Cambridge Univ. Press), IAU Colloq., 111, 103

Schlegel, D. J., Finkbeiner, D. P., \& Davis, M. 1998, ApJ, 500, 525

Smith, H. A. 1995, RR Lyrae Stars (Cambridge: Cambridge Univ. Press)

Sneden, C., Kraft, R. P., Guhathakurta, P., Peterson, R. C., \& Fulbright, J. P. 2004, AJ, 127, 2162

Szeidl, B. 1973, Comm. Konk. Obs., 63, 1

VandenBerg, D. A., \& Clem, J. L. 2003, AJ, 126, 778 